\title{
Solar-like oscillations in open cluster stars
}

\author{
D. Stello, ${ }^{1}$ H. Bruntt, ${ }^{1}$ T. Arentoft, ${ }^{2,3}$ R. L. Gilliland, ${ }^{4}$ J. Nuspl, ${ }^{5}$ S.-L. Kim, ${ }^{6}$ Y. B. Kang, ${ }^{6}$
} J.-R. Koo, ${ }^{6}$ J.-A. Lee, ${ }^{6}$ C.-U. Lee, ${ }^{6}$ C. Sterken, ${ }^{7}$ A. P. Jacob, ${ }^{1}$ S. Frandsen, ${ }^{2,3}$ Z. E. Dind, ${ }^{1}$ H. R. Jensen, ${ }^{2}$ R. Szabó, ${ }^{5}$ Z. Csubry, ${ }^{5}$ L. L. Kiss, ${ }^{1}$ M. Y. Bouzid, ${ }^{7}$ T. H. Dall, ${ }^{8}$

T. R. Bedding, ${ }^{1}$ H. Kjeldsen ${ }^{2,3}$

\author{
${ }^{1}$ School of Physics, University of Sydney, NSW 2006, Australia \\ ${ }^{2}$ Institut for Fysik og Astronomi (IFA), Aarhus Universitet, 8000 Aarhus, Denmark \\ ${ }^{3}$ Danish AsteroSeismology Centre, Aarhus Universitet, 8000 Aarhus, Denmark \\ ${ }^{4}$ Space Telescope Science Institute, 3700 San Martin Dr., Baltimore, USA \\ ${ }^{5}$ Konkoly Observatory, 1525 Budapest, PO Box 67, Hungary \\ ${ }^{6}$ Korea Astronomy and Space Science Institute, Daejeon 305-348, Korea \\ ${ }^{7}$ Vrije Universiteit Brussel, Pleinlaan 2, B-1050 Brussels, Belgium \\ ${ }^{8}$ European Southern Observatory, Casilla 19001, Santiago 19, Chile
}

\section{Introduction}

Asteroseismology of stellar clusters is potentially a powerful tool. The assumption of a common age, distance, and chemical composition provides constraints on each cluster member, which significantly improves the asteroseismic output. Hence, detecting oscillations in cluster stars in a range of evolutionary stages holds promise of providing more stringent tests of stellar evolution theory. Driven by this great potential, we carried out multi-site observations aimed at detecting solar-like oscillations in the red giant stars in the open cluster M67 (NGC 2682). To obtain these data we observed for 43 days with nine $0.6-\mathrm{m}$ to $2.1-\mathrm{m}$ class telescopes in January and February 2004 (Stello et al. 2006). The photometric time series comprises roughly 18000 data points. The properties of the red giant stars we present here are given in Table 1 and their location in the colour-magnitude diagram is shown in Fig. 1 (left panel).
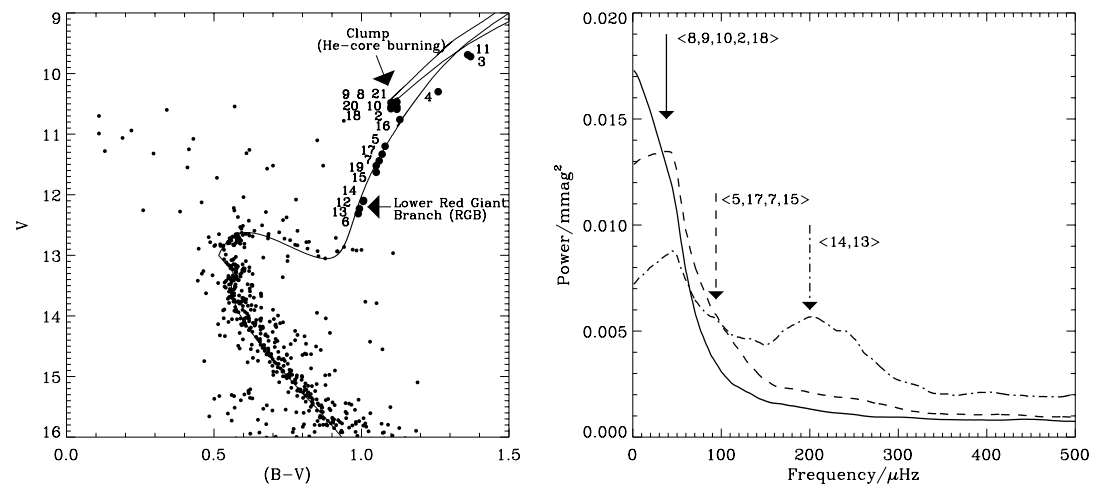

Figure 1: Left Panel: The colour-magnitude diagram of the open cluster M67. The red giant target stars and their ID are indicated. Right Panel: Average power distributions for three groups of stars sorted according to luminosity: most luminous (clump stars), intermediate, and least luminous (lower RGB). Arrows show expected locations of solar-like oscillations (see Table 1). Only stars with a white-noise level lower than $50 \mu \mathrm{mag}$ have been used. 
Table 1: Properties of red giant target stars. Luminosities and temperatures are from photometry (Montgomery et al. 1993). Estimates of oscillation amplitudes, characteristic frequencies and large separations are from known scaling relations in the literature (Kjeldsen \& Bedding 1995, Brown et al. 1991). Crossreferences are to Sanders (1977) and Gilliland et al. (1991).

\begin{tabular}{rrrrrrl}
\hline \hline ID & $L / L_{\odot}$ & $\begin{array}{r}T_{\text {eff }} \\
\mathrm{K}\end{array}$ & $\begin{array}{r}\delta L / L \\
\mu \mathrm{mag}\end{array}$ & $\begin{array}{r}\nu_{\max } \\
\mu \mathrm{Hz}\end{array}$ & $\begin{array}{c}\Delta \nu_{0} \\
\mu \mathrm{Hz}\end{array}$ & Cross-ref. \\
\hline \hline 8 & 50.8 & 4750 & 287 & 35.8 & 4.3 & $\mathrm{~S} 1010 / \mathrm{G} 2$ \\
9 & 50.2 & 4772 & 281 & 36.8 & 4.4 & $\mathrm{~S} 1084 /-$ \\
10 & 48.2 & 4727 & 275 & 37.0 & 4.4 & $\mathrm{~S} 1279 / \mathrm{G} 7$ \\
2 & 46.4 & 4727 & 265 & 38.4 & 4.6 & $\mathrm{~S} 1074 /-$ \\
18 & 45.8 & 4772 & 256 & 40.3 & 4.7 & $\mathrm{~S} 1316 /-$ \\
\hline 5 & 25.4 & 4815 & 140 & 74.8 & 7.6 & $\mathrm{~S} 1054 / \mathrm{G} 9$ \\
17 & 22.4 & 4835 & 122 & 86.0 & 8.4 & $\mathrm{~S} 1288 /-$ \\
7 & 20.2 & 4854 & 109 & 96.9 & 9.2 & $\mathrm{~S} 989 / \mathrm{G} 12$ \\
15 & 16.9 & 4873 & 91 & 117.3 & 10.6 & $\mathrm{~S} 1277 /-$ \\
\hline 14 & 11.2 & 4945 & 58 & 187.3 & 15.2 & $\mathrm{~S} 1293 /-$ \\
13 & 9.9 & 4966 & 51 & 213.2 & 16.8 & $\mathrm{~S} 1305 /-$ \\
\hline \hline
\end{tabular}

\section{Results}

Mean levels in the Fourier spectra in the frequency interval $300-900 \mu \mathrm{Hz}$, corresponding to white noise, reach $20 \mu \mathrm{mag}$ for the stars with the lowest noise. In many stars we see apparently high levels of non-white noise, but the detailed temporal variation of the noise is unknown. We are therefore not able to clearly disentangle the noise and stellar signal in the analysis. However, we do see evidence of excess power in the Fourier spectra, shifting to lower frequencies for more luminous stars, consistent with expectations (Fig. 1; right panel). If the observed power excesses were due to stellar oscillations, this result would show great prospects for asteroseismology in stellar clusters. A more detailed analysis will be given by Stello et al. (2007).

Acknowledgments. This paper has been supported by the Astronomical Society of Australia.

\section{References}

Brown T. M., Gilliland R. L., Noyes R. W., Ramsey L. W., 1991, ApJ, 368, 599

Gilliland R. L., Brown T. M., Thomson D. T., et al., 1991, AJ, 101, 541

Kjeldsen H., Bedding T. R., 1995, A\&A, 293, 87

Montgomery K. A., Marschall L. A., Janes K. A., 1993, AJ, 106, 181

Sanders W. L., 1977, A\&AS, 27, 89

Stello D., Arentoft T., Bedding T. R., et al., 2006, MNRAS, 373, 1141

Stello D., Bruntt H., Kjeldsen H., et al., 2007, MNRAS, in press 